\title{
Revisão sistemática e metanálise do uso de procinéticos no refluxo gastroesofágico e na doença do refluxo gastroesofágico em Pediatria
}

\author{
Systematic review and meta-analysis of the use of prokinetics in gastroesophageal reflux \\ and in gastroesophageal reflux disease in Pediatrics
}

Pedro Vieira S. Magalhães ${ }^{1}$, Telma Regina P. D. Bastos², José Carlos B. Appolinário ${ }^{3}$, Josué Bacaltchuk ${ }^{4}$, Joaquim Ignácio S. Mota Neto ${ }^{5}$

\section{RESUMO}

Objetivo: Avaliar, por meio de revisão sistemática e metanálise, estudos randomizados que comparam os procinéticos domperidona, bromoprida, metoclopramida e betanecol ao placebo no tratamento do refluxo gastroesofágico (RGE) e da doença do refluxo gastroesofágico (DRGE) em crianças.

Métodos: Busca bibliográfica de ensaios clínicos randomizados (Medline, EMBASE, Biological Abstracts, ISI/Web of Science, CINAHL, Lilacs e Cochrane). O desfecho primário foi eficácia na modificação dos sintomas de refluxo, conforme definição de autores das fontes primárias. Outras variáveis de interesse foram: complicações relacionadas ao RGE, alterações nos exames laboratoriais de controle, qualidade de vida, eventos adversos e abandono do tratamento.

Resultados: Foram incluídos quatro estudos com domperidona, dois com metoclopramida, um com betanecol. Nenhum estudo com bromoprida foi localizado. O risco de não resposta ao tratamento foi significativamente menor para os procinéticos quando comparados ao placebo ( $R R$ 0,35; IC95\% 0,14-0,88). A vantagem terapêutica individual em relação ao placebo se manteve para a domperidona $(n=126$; RR 0,27; IC95\% 0,14-0,52; NNT 3; I 0\%) e betanecol ( $\mathrm{n}=44, \mathrm{RR} 0,19$, IC95\% 0,05-0,55, NNT 2), mas não para metoclopramida ( $\mathrm{n}=71$; RR 0,63; IC95\% 0,07-5,71, $\left.\mathrm{I}_{2}, 92,2 \%\right)$.

Conclusões: A evidência para o uso de procinéticos no RGE e na DRGE em crianças é limitada, pois os poucos estudos são ensaios preliminares de resposta em curto prazo e com limitações metodológicas.

Palavras-chave: metanálise; refluxo gastroesofágico; metoclopramida; domperidona; betanecol; Pediatria.

\section{ABSTRACT}

Objective: To evaluate, by systematic review and metaanalysis, randomized studies comparing the prokinetics (domperidone, bromopride, metoclopramide and bethanechol) to placebo in the treatment of gastroesophagic reflux (GER) and gastroesophagic reflux disease (GERD) in children.

Methods: Bibliographic search for randomized clinical trials (Medline, EMBASE, Biological Abstracts, ISI/Web of Science, CINAHL, Lilacs e Cochrane). The primary outcome was the modification of reflux symptoms. Other outcomes were: GER-related complications, alterations in control exams, life quality, adverse events and abandon of treatment.

Results: The metanalysis included four studies on domperidone, two on metoclopramide, and one on bethane-
Instituição: Centro de Medicina Baseada em Evidências da Universidade Federal de Pelotas (UFPel), Pelotas, RS, Brasil

${ }^{1}$ Mestre em Saúde e Comportamento pela Universidade Católica de Pelotas (UCPel); pesquisador do Centro de Medicina Baseada em Evidências da UFPel e do Instituto Nacional de Ciência e Tecnologia Translacional em Medicina do Hospital de Clínicas de Porto Alegre, Porto Alegre, RS, Brasil

${ }^{2}$ Doutora em Medicina pela Universidade de São Paulo (USP), São Paulo, SP, Brasil

${ }^{3}$ Doutor em Medicina pela Universidade Federal do Rio de Janeiro (UFRJ); médico do Instituto de Psiquiatria da UFRJ, Rio de Janeiro, RJ, Brasil ${ }^{4}$ Doutor em Medicina pela Universidade Federal de São Paulo (Unifesp); Médico do Departamento de Psiquiatria da Unifesp, São Paulo, SP, Brasil ${ }^{5}$ Mestre em Saúde e Comportamento pela UCpel; pesquisador do Centro de Medicina Baseada em Evidências da UFPel, Pelotas, RS, Brasil

Endereço para correspondência:
Pedro V. S. Magalhães

Avenida Independência, 44/905 - Centro

CEP 90035070 - Porto Alegre/RS

E-mail: pedromaga2@gmail.com

Declaração de conflito de interesse: Pedro Vieira S. Magalhães e Joaquim Ignácio S. Mota Neto receberam financiamento da Janssen-Cilag Farmacêutica; Telma Regina P. D. Bastos, José Carlos B. Appolinário e Josué Bacaltchuk trabalham na Janssen-Cilag Farmacêutica.

Fonte financiadora: Janssen-Cilag Farmacêutica

Recebido em: 03/7/08

Aprovado em: 24/1/09 
chol. No study of bromopride was retrieved. The risk of non-response to the treatment was significantly smaller in children that received prokinetics in comparison to placebo (RR 0.35, 95\% CI 0.14-0.88). Individual therapeutic advantage regarding placebo was related to domperidone $\left(\mathrm{n}=126\right.$; RR 0.27; 95\%CI 0.14-0.52, NNT 3; $\left.\mathrm{I}_{2} 0 \%\right)$ and bethanechol ( $\mathrm{n}=44$; RR 0.19; 95\%CI 0.05-0.55; NNT 2), but not to metoclopramide ( $\mathrm{n}=71$; RR 0.63 ; 95\% CI 0.075.71; I $92.2 \%)$.

Conclusions: The evidence for prokinetic use in GER and GERD in children is limited because the few studies report preliminary trials that evaluate short-term responses and show methodological limitations.

Key-words: meta-analysis; gastroesophageal reflux; metoclopramide; domperidone; bethanechol; Pediatrics.

\section{Introdução}

O refluxo gastroesofágico (RGE) é definido como o retorno passivo do conteúdo gástrico para o esôfago, independentemente de sua etiologia ${ }^{(1-3)}$. Tal fenômeno pode ocorrer em circunstâncias fisiológicas ou patológicas e em qualquer indivíduo, seja criança ou adulto. Quando não está associado a doenças ou complicações, é denominado RGE fisiológico. O RGE patológico, ou doença do refluxo gastroesofágico (DRGE), é uma condição menos comum e possui prognóstico mais grave, além de abordagens diagnóstica e terapêutica diferentes ${ }^{(4)}$. Os sintomas presentes da DRGE pediátrica incluem: regurgitações e/ou vômitos, irritabilidade, choro excessivo, anorexia e recusa alimentar, entre outros. Em crianças de faixa etária mais avançada, são observados sintomas semelhantes aos encontrados em adultos, como pirose e regurgitação crônica ou manifestações extraesofagianas.

O Working Group on Gastro-oesophageal Reflux Disease da Sociedade Europeia de Gastroenterologia, Hepatologia e Nutrição Pediátrica (ESPGHAN) propõe a diferenciação entre RGE fisiológico e DRGE com base nos achados clínicos e na resposta à terapêutica ${ }^{(1)}$. A recomendação para o manejo do RGE/DRGE segundo o ESPHGAN e o North American Society for Pediatric Gastroenterology and Nutrition (NASPGN) consiste no emprego de medidas conservadoras tais como: orientação aos pais, tratamento postural e medidas dietéticas. Nos casos em que essas medidas forem insuficientes, indica-se o teste terapêutico com um procinético ${ }^{(3)}$.

No manejo do RGE e da DRGE, procinéticos têm sido usados há muitos anos. São drogas que estimulam a motili- dade do aparelho digestivo por ação direta sobre o músculo liso entérico ou por interação com os neurônios do sistema nervoso entérico ${ }^{(5)}$. Avaliações críticas dos relatos publicados sobre a eficácia dos diferentes procinéticos no RGE/DRGE em crianças (cisaprida, domperidona e metoclopramida) concluíram que a cisaprida foi a mais eficaz ${ }^{(6-8)}$. Porém, devido à indisponibilidade da cisaprida, outros agentes procinéticos passaram a ser utilizados. As mais recentes revisões sobre o RGE/DRGE em Pediatria são narrativas e não sistemáticas ${ }^{(4,9,10)}$ ou avaliam uma única droga ${ }^{(11,12)}$. Desta forma, o presente estudo tem por objetivo avaliar a eficácia e a segurança, de forma abrangente, por revisão sistemática e metanálise dos estudos randomizados que comparam os procinéticos domperidona, bromoprida, metoclopramida e betanecol ao placebo no tratamento do refluxo gastroesofágico (RGE) e da doença do refluxo gastroesofágico (DRGE) em crianças.

\section{Métodos}

Foram incluídos estudos randomizados relevantes, com período de acompanhamento de pelo menos uma semana. Como o nível de mascaramento não foi definitivamente ligado a viés ${ }^{(13)}$, estudos simples-cegos foram analisados. Ensaios do tipo cross-over foram também incluídos, mas apenas dados do período inicial paralelo foram analisados.

Os estudos deveriam incluir primariamente crianças até 12 anos de idade com RGE ou DRGE, de acordo com definição dos autores (por critérios clínicos, pHmetria etc.). Os pacientes poderiam ser recrutados em qualquer nível de atendimento (pacientes externos e internos). Estudos que avaliaram o impacto do tratamento antirrefluxo em relação a comorbidades relevantes associadas a RGE/DRGE também foram incluídos.

Foram avaliados, nesta revisão, apenas estudos sobre drogas disponíveis correntemente para o tratamento de RGE/ DRGE (domperidona, bromoprida, metoclopramida, betanecol) em comparação a um placebo inerte; estudos (ou braço de estudos) comparando intervenções combinadas (mais de uma intervenção simultânea) com placebo foram excluídos.

Nesta revisão, o interesse primário foi a modificação nos sintomas do refluxo. A partir de revisões prévias da literatura, antecipou-se a existência de relativamente poucos estudos. Logo, a definição de "modificação de sintomas" foi propositalmente mantida ampla e de acordo com a definição original dos estudos primários. Assim, poderiam ser incluídos estudos que utilizassem classificações de resposta que compreendessem algum tipo de avaliação clínica, como 
redução na intensidade ou frequência de sintomas, mesmo que conjuntamente com parâmetros laboratoriais. Nos resultados, apresenta-se o risco de ausência de resposta. Este risco é o inverso da probabilidade de resposta. Logo, um risco de não resposta de 0,5 equivale a uma probabilidade duas vezes maior de resposta clínica.

Outros resultados de interesse foram: complicações relacionadas a RGE/DRGE (como hospitalizações, baixo ganho de peso, sintomas respiratórios, esofagite etc.); resultados de pHmetria, alterações histológicas e resultados de manometria esofágica; eventos adversos relacionados ao tratamento; qualidade de vida/atividade; satisfação com o tratamento e custo-efetividade.

Quando os estudos apresentaram resultados de sintomas como categorias múltiplas, os resultados foram dicotomizados como resposta/ausência de resposta, considerando-se resposta pelo menos alguma modificação nos sintomas.

As buscas foram conduzidas nas seguintes bases de dados: Medline (1966-2006), EMBASE (1974-2006), Biological Abstracts, ISI/Web of Science, CINAHL, Lilacs e Cochrane Central Register of Controlled Trials. Além disso, artigos localizados, consensos e revisões foram também examinados para quaisquer outras referências adicionais. Dados não publicados não foram especificamente buscados, mas, quando citados na literatura localizada, foram também analisados. Poderiam ser incluídos, a princípio, artigos em qualquer idioma; no caso em questão, entretanto, apenas trabalhos de língua inglesa foram encontrados.

A estratégia de busca combinou os termos em inglês: gastro-oesophageal reflux OR gastro-esophageal reflux OR gerd OR gord OR gastro-esophageal reflux disease OR gastrooesophageal reflux disease OR oesophagitis OR esophagitis OR regurgitation) AND (child OR children OR infant* OR pediatric OR newborn) AND (random* OR control* OR versus OR placebo OR cross-over OR crossover OR factorial) AND (bethanechol OR metoclopramide OR bromopride OR domperidone).

Uma forma padrão delineada pelos autores foi usada para extrair características e resultados dos estudos triados. Ocultamento de alocação, de acordo com o critério do Cochrane Collaboration Reviewer's Handbook ${ }^{(14)}$, foi a forma primária de avaliação de qualidade. Estudos com forma inadequada de ocultamento de alocação foram excluídos da análise final. A escala Jadad ${ }^{(15)}$ foi usada para avaliar a qualidade dos estudos. Tal escala avalia três itens: nível de cegamento, ocultamento de alocação e descrição de perdas. Sua pontuação varia de zero a 5 .
Apesar de a escolha do modelo de metanálise permanecer controversa $^{(16)}$, empregou-se o modelo de efeitos aleatórios ${ }^{(17)}$, que assume que estudos analisados atualmente provêm de um pool de estudos hipotéticos. Foram utilizados riscos relativos para sumariar dados, desde que fossem fáceis de interpretar ${ }^{(16)}$. Para medidas contínuas, a diferença média padronizada, que resulta em uma estimativa de tamanho de efeito, foi empregada. Quando os resultados originados de análises por intenção de tratar estavam disponíveis a partir dos estudos originais, foram preferidos.

O gráfico em funil foi utilizado com a finalidade de avaliar a existência de viés nas publicações selecionadas. Além de usar um teste estatístico para checar a heterogeneidade ${ }^{(18)}$, foram planejadas várias análises de subgrupos para investigar diferenças de tratamento relevantes. Os grupos decididos a priori para análise de subgrupo foram: (1) método de diagnóstico, especialmente em relação à exclusão de outros diagnósticos, como intolerância à lactose ${ }^{(19)}$; (2) idade do participante (menor de seis meses, de seis a 12 meses, maior de 12 meses); (3) duração do acompanhamento; (4) local do tratamento e (5) qualidade do estudo.

\section{Resultados}

Na metanálise, foram incluídos quatro estudos com domperidona, dois com metoclopramida e um que avaliou o betanecol. Não foram localizados estudos com a bromoprida. Os estudos se restringiram a avaliar a resposta clínica em curto prazo e medidas laboratoriais. Como pode ser observado na Tabela 1, os estudos foram tipicamente pequenos, incluindo pacientes com variação de idade ampla, com acompanhamento médio curto (quatro semanas) e resultados geralmente limitados ao sintoma e medidas laboratoriais. A qualidade dos relatos foi uniformemente moderada, com um escore de Jadad mediano de 3 . Nenhum dos estudos incluídos referiu como realizou o ocultamento de alocação.

Em relação à análise principal sobre a modificação de sintomas de refluxo, foram encontrados seis estudos ${ }^{(20-25)}$. Como é possível observar na Tabela 1 , a definição de melhora nesses estudos abrangia medida de sintomas avaliada pelo investigador em conjunção ou não a critérios laboratoriais, como sondagem de $\mathrm{pH}$ ou endoscopia. A metanálise indicou um risco menor de não melhora ao final do tratamento para os procinéticos em relação ao placebo (RR 0,35; IC95\% $0,14-0,88, p=0,02)$, mas com importante heterogeneidade $\left(\mathrm{I}_{2} 72,2 \%\right)$. Em relação às medicações individuais, como observado na Figura 1, a vantagem em relação ao placebo se 
Tabela 1 - Características dos ensaios clínicos randomizados que compararam procinéticos ao placebo e forneceram dados em relação à modificação de sintomas de refluxo

\begin{tabular}{|c|c|c|c|c|}
\hline Estudo & Intervenções & Participantes & Métodos & $\begin{array}{l}\text { Resultados } \\
\text { disponíveis }\end{array}$ \\
\hline $\begin{array}{l}\text { Bellissant } \\
\text { et } a^{(20)}\end{array}$ & $\begin{array}{l}\text { Metoclopramida }(0,2 \mathrm{mg} / \mathrm{kg} \\
\text { TID) versus placebo } \\
\text { Cointervenção: terapia } \\
\text { postural }\end{array}$ & $\begin{array}{l}\text { Idade: } 4 \text { sem } 8 \text { m }(n=44) \\
\text { Diagnóstico: RGE (pHmetria de 24h) } \\
\text { Exclusão: esofagite, estenose } \\
\text { esofagiana, hérnia, úlcera, cirurgia } \\
\text { antirrefluxo, tratamento prévio }\end{array}$ & $\begin{array}{l}\text { Local: incerto } \\
\text { Acompanhamento: } \\
2 \text { semanas } \\
\text { Análise não ITT } \\
\text { Jadad: } 3\end{array}$ & $\begin{array}{l}\text { Medidas de pH } \\
\text { Peso } \\
\text { Avaliação dos } \\
\text { pais }\end{array}$ \\
\hline $\begin{array}{l}\text { Carroccio } \\
\text { et } a^{(21)}\end{array}$ & $\begin{array}{l}\text { Domperidona }(0,3 \mathrm{mg} / \mathrm{kg} \\
\text { TID) versus placebo } \\
\text { Cointervenção: alimentos } \\
\text { engrossados }\end{array}$ & $\begin{array}{l}\text { Idade: } 1-18 \text { m ( } \mathrm{n}=40) \\
\text { Diagnóstico: RGE (pHmetria de 24h) } \\
\text { Exclusão: incerta, mas DRGE } \\
\text { permitida }\end{array}$ & $\begin{array}{l}\text { Pacientes } \\
\text { externos } \\
\text { Acompanhamento: } \\
8 \text { semanas } \\
\text { Análise: incerta } \\
\text { Jadad: } 2\end{array}$ & $\begin{array}{l}\text { Melhora: } \\
\text { combinação } \\
\text { de sintomas e } \\
\text { sondagem de pH }\end{array}$ \\
\hline Clara $^{(22)}$ & $\begin{array}{l}\text { Domperidona }(0,3 \mathrm{mg} / \mathrm{kg} \\
\text { TID) versus placebo } \\
\text { Cointervenção: medidas } \\
\text { dietéticas }\end{array}$ & $\begin{array}{l}\text { Idade: } 2 \text { sem - } 10 \text { anos }(n=32) \\
\text { Diagnóstico: regurgitação crônica } \\
\text { excessiva com incapacidade do EEI } \\
\text { Exclusão: incerta }\end{array}$ & $\begin{array}{l}\text { Pacientes } \\
\text { externos } \\
\text { Acompanhamento: } \\
4 \text { semanas } \\
\text { Análise ITT } \\
\text { Jadad: } 3\end{array}$ & $\begin{array}{l}\text { Melhora avaliada } \\
\text { pelo investigador }\end{array}$ \\
\hline $\begin{array}{l}\text { Cucchiara } \\
\text { et } a^{(23)}\end{array}$ & $\begin{array}{l}\text { Domperidona }(0,3 \mathrm{mg} / \mathrm{kg} \\
\text { TID) versus placebo } \\
\text { Cointervenção: terapia } \\
\text { postural, alimentos } \\
\text { engrossados }\end{array}$ & $\begin{array}{l}\text { Idade: } 2 \text { m-13 m ( } n=26) \\
\text { Diagnóstico: esofagite de refluxo } \\
\text { Exclusão: esofagite severa }\end{array}$ & $\begin{array}{l}\text { Local: incerto } \\
\text { Acompanhamento: } \\
12 \text { semanas } \\
\text { Análise incerta } \\
\text { Jadad: } 2\end{array}$ & $\begin{array}{l}\text { Melhora: } \\
\text { combinação de } \\
\text { avaliação clínica } \\
\text { e endoscopia }\end{array}$ \\
\hline $\begin{array}{l}\text { De Loore } \\
\text { et } a^{(24)}\end{array}$ & $\begin{array}{l}\text { Domperidona } \\
(0,3 \mathrm{mg} / \mathrm{kg} \text { TID) versus } \\
\text { Metoclopramida }(0,3 \mathrm{mg} / \mathrm{kg} \\
\text { TID) versus placebo } \\
\text { Cointervenção: incerta }\end{array}$ & $\begin{array}{l}\text { Idade: } 3 \text { sem-8 anos }(n=47) \\
\text { Diagnóstico: regurgitação excessiva } \\
\text { Exclusão: lesões orgânicas }\end{array}$ & $\begin{array}{l}\text { Pacientes } \\
\text { externos } \\
\text { Acompanhamento: } \\
2 \text { semanas } \\
\text { Análise: incerta }\end{array}$ & $\begin{array}{l}\text { Melhora avaliada } \\
\text { pelo investigador }\end{array}$ \\
\hline Euler ${ }^{(25)}$ & $\begin{array}{l}\text { Betanecol }\left(8,7 \mathrm{mg} / \mathrm{m}^{2} \mathrm{TID}\right) \\
\text { versus placebo } \\
\text { Cointervenção: terapia } \\
\text { postural }\end{array}$ & $\begin{array}{l}\text { Idade: } 3 \text { sem- } 13 \text { anos }(n=45) \\
\text { Diagnóstico: RGE (pHmetria de } 24 \mathrm{~h}) \\
\text { Exclusão: incerta }\end{array}$ & $\begin{array}{l}\text { Pacientes } \\
\text { externos } \\
\text { Acompanhamento: } \\
6 \text { semanas } \\
\text { Análise ITT } \\
\text { Jadad: } 3\end{array}$ & $\begin{array}{l}\text { Melhora clínica } \\
\text { avaliada pelo } \\
\text { investigador } \\
\text { Descontinuação }\end{array}$ \\
\hline
\end{tabular}

RGE: refluxo gastroesofágico; DRGE: doença do refluxo gastroesofágico; ITT: intenção de tratar; EEl: esfíncter esofagiano inferior.

manteve para a domperidona ( $\mathrm{n}=126$; RR 0,27; IC95\% 0,14$0.52 ; p<0,001$; NNT $\left.3 ; \mathrm{I}_{2} 0 \%\right)$ e para o betanecol $(\mathrm{n}=44$, RR 0,19; IC95\% 0,05-0,55; $p=0,002$; NNT 2), mas não para a metoclopramida ( $\mathrm{n}=71$, RR 0,63, IC95\% 0,07-5,71; $\left.p=0,68, \mathrm{I}_{2} 92,2 \%\right)$. Quando analisadas as drogas individualmente (Tabela 2), foi possível observar a heterogeneidade nos estudos com metoclopramida ${ }^{(20)}$. Um ensaio comparou ainda o escore clínico médio no término ${ }^{(23)}$, sendo que a domperidona não foi diferente do placebo nesta comparação (SMD 0,14; IC95\% -0,63 a 0,91; $p=0,72$ ).

Apenas um estudo expôs dados a respeito do peso das crianças no término do tratamento ${ }^{(20)}$. O peso final não diferiu em pacientes tratados com metoclopramida comparados ao grupo placebo (SMD -0,16; IC95\% -0,79 a 0,47; $p=0,61$ ).

Nenhuma das medidas de pHmetria ao término diferiu em estudos com metoclopramida ${ }^{(20)}$. Em relação à domperidona, o 


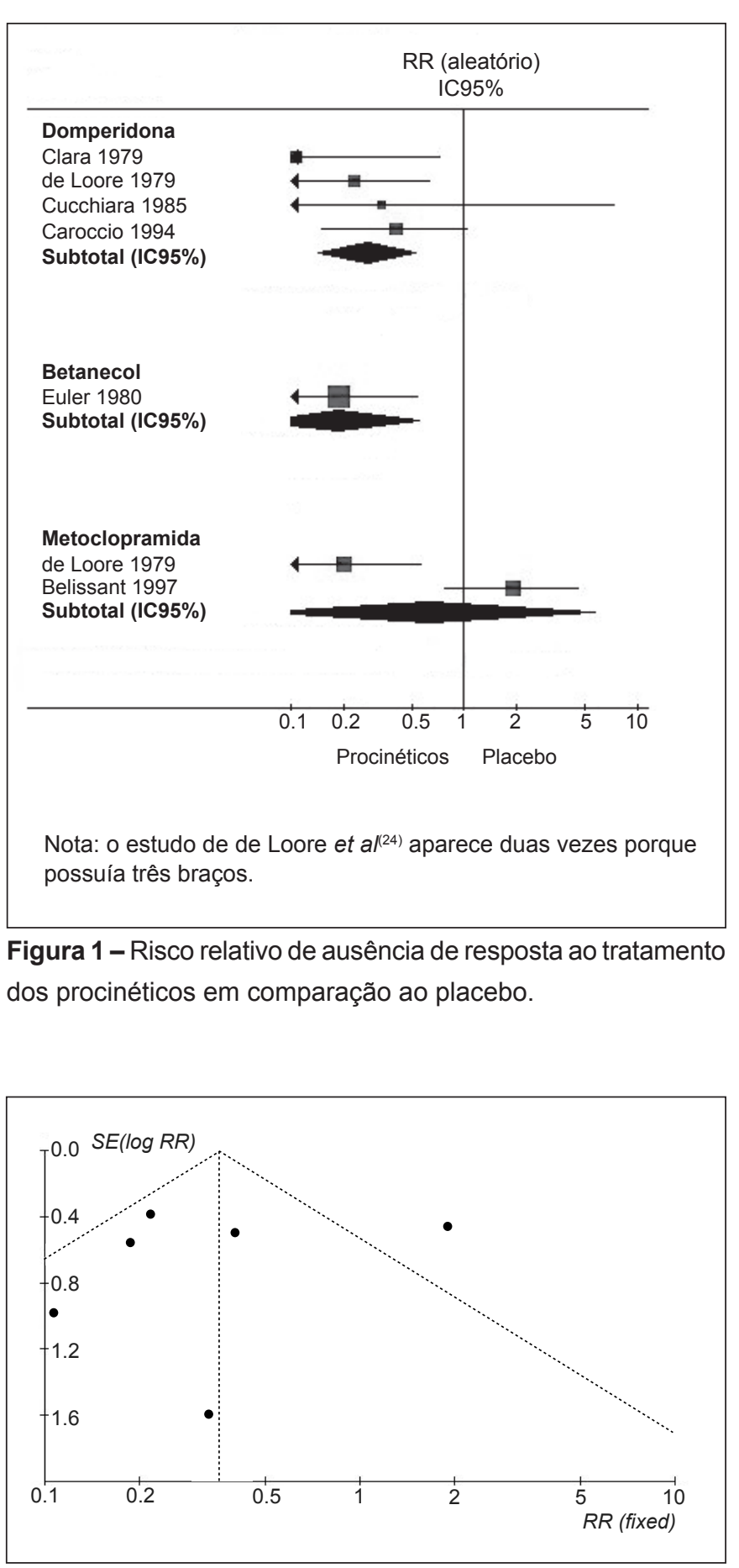

Figura 2 - Gráfico em funil de procinéticos versus placebo. risco de se ter menos do que $25 \%$ de diminuição de episódios de refluxo chegou próximo à significância em favor da droga em um estudo (RR 0,08; IC95\% 0,01-1,12; $p=0,06)^{(26)}$. Além disso, não houve diferença no esvaziamento gástrico no término (RR 0,57, IC95\% 0,22-1.17; $p=0,25$ ). Em outro estudo ${ }^{(23)}$ que comparou a domperidona e o placebo por meio de um sistema de escore de esofagite, não houve diferença ao término do tratamento (SMD 0,11; IC95\% -0,66 a 0,88; $p=0,78$ ).

Como um todo, pacientes que receberam procinéticos foram estatisticamente mais propensos a desistir dos estudos $^{(20,25-27)}$ em comparação ao placebo ( $\mathrm{n}=114 ; \mathrm{RR} 3,24$; IC95\% 1,06-9.92; $p=0,04$, NNH 6; $\mathrm{I}_{2}=0 \%$ ). Um estudo forneceu dados apenas relativos a uma crise oculógira sofrida por um paciente enquanto se tratava com metoclopramida ${ }^{(28)}$.

Embora poucos estudos tenham sido incluídos, não foi possível inferir viés de publicação pelo gráfico em funil (Figura 2).

\section{Discussão}

Vários fármacos fazem parte do arsenal terapêutico disponível na pediatria clínica para o tratamento do RGE/DRGE, entre eles procinéticos e antissecretores ${ }^{(29)}$. No entanto, verifica-se que seu uso não é baseado em evidência científica consistente. Um fato que chamou a atenção dos autores foi a inexistência, dentro da metodologia proposta, de estudos clínicos realizados com a bromoprida, apesar de seu uso frequente na clínica pediátrica no Brasil.

Considerando que a DRGE pode evoluir com complicações de gravidade variável ao longo de seu curso clínico, torna-se necessário avaliar os dados científicos acerca das intervenções farmacológicas e não farmacológicas disponíveis. Embora alguns relatos favoreçam o uso de intervenções não farmacológicas no RGE/DRGE, uma revisão sistemática recente não encontrou evidência confiável sobre a eficácia de procedimentos conservadores como medidas posturais, espessamento das refeições e alterações em fórmulas ${ }^{(30)}$. Este fato afirma a necessidade de se abordarem diversas intervenções no RGE/DRGE pediátrico.

Tabela 2 - Procinéticos e risco de não ter resposta clínica de curto prazo (comparados ao placebo)

\begin{tabular}{lcccc}
\hline Comparação & $\mathbf{n}$ & RR (IC 95\%) & Heterogeneidade (I2) & NNT \\
\hline Betanecol & 44 & $0,19(0,05-0,55)$ & n/a & 2 \\
Domperidona & 128 & $0,27(0,14-0,52)$ & zero & 3 \\
Metoclopramida & 71 & $0,63(0,07-5,71)$ & $92 \%$ & n/a \\
\hline
\end{tabular}

RR: risco relativo; IC: intervalo de confiança; NNT: número necessário para tratar; n/a: não avaliado. 
Nesta revisão sistemática, observou-se que os procinéticos, em geral, promoveram melhora dos sintomas em curto prazo quando comparados ao placebo. Os estudos realizados com domperidona mostraram resultados mais consistentes, enquanto aqueles realizados com outros agentes demonstraram resultados inconclusivos, já que poucos pacientes foram randomizados para o uso de metoclopramida e um número menor ainda para betanecol, este último avaliado em apenas um estudo. Além de amplamente ineficaz em um dos estudos ${ }^{(20)}$, a eficácia da metoclopramida não foi satisfatória devido a problemas de recrutamento em outro estudo ${ }^{(27)}$. Neste último, nenhum dos três pacientes randomizados para receber tratamento ativo completaram o estudo devido a questões relacionadas à tolerabilidade da droga, o que é consistente com a farmacologia da metoclopramida no Sistema Nervoso Central (SNC) $)^{(4)}$.

Devido à presença de receptores dopaminérgicos no SNC, reações adversas relacionadas à metoclopramida podem incluir agitação, sonolência e sintomas extrapiramidais, como distonias e discinesias ${ }^{(4,9)}$. Apesar de não serem observadas reações adversas significativas com os demais procinéticos nos estudos incluídos na presente revisão, sabe-se da potencial associação de cada um deles a determinados eventos. O uso da bromoprida ${ }^{(31)}$ e da domperidona ${ }^{(11)}$ pode levar à ocorrência de eventos extrapiramidais, principalmente em recém-nascidos prematuros e lactentes jovens devido à imaturidade do sistema nervoso e da barreira hematoencefálica ${ }^{(31,11)}$. Todavia, tais eventos não são frequentemente associados ao uso da domperidona, já que esta substância possui elevado peso molecular e limitada passagem através da barreira hematoencefálica ${ }^{(32)}$. Por sua ação em receptores colinérgicos, o betanecol pode provocar broncoconstrição e hipotensão, entre outros efeitos ${ }^{(9)}$. Conforme mencionado anteriormente, optou-se por não incluir a cisaprida em virtude de sua atual indisponibilidade na maioria dos países. Prolongamento do intervalo QT e arritmias cardíacas foram potencialmente relacionados ao seu uso em pacientes pediátricos ${ }^{(4,9)}$. Eventos cardíacos podem ocorrer também com o uso da domperidona, sobretudo se associada a drogas metabolizadas pela CTP3A4, como alguns antifúngicos ${ }^{(11)}$. Fatores familiares e genéticos são apontados como contribuintes para a ocorrência de tais eventos ${ }^{(33)}$.

Dentre as limitações encontradas nos estudos avaliados, destacam-se aquelas referentes aos desfechos avaliados: da escolha de parâmetros (clínicos e/ou laboratoriais) aos métodos para avaliação de tais parâmetros. Dessa forma, encontram-se como desfechos para o uso de procinéticos sintomas clínicos diversos, instrumentos discrepantes para abordar sintomas, dificuldade em descartar alergia ao leite de vaca e limitações para avaliação de outras cointervenções.
Além disso, os ensaios clínicos não avaliaram respostas em longo prazo. Os estudos cujas respostas se basearam em resultados de $\mathrm{pHmetria}$ ou $\mathrm{pH}$-impedanciomentria, com relevância crescente em pediatria, foram escassos. Observase, ainda, importante heterogeneidade relacionada à faixa etária das crianças estudadas, o que pode ter impacto na definição do tipo de refluxo e na resposta terapêutica, entre outros fatores. Apesar dos objetivos propostos pelo presente estudo, não foram encontrados, em nenhum dos estudos analisados, dados referentes a impacto da medicação na qualidade de vida, redução de complicações ou análise de custo-eficácia das intervenções utilizadas. A quantidade de estudos foi limitada e o número de crianças randomizadas muito pequeno para um problema clínico tão relevante; dessa forma, não foi possível efetuar a análise proposta em todos os subgrupos.

Estudos futuros deverão não apenas controlar as cointervenções, mas também estabelecer claramente o tipo de refluxo, pré-definir desfechos clínicos e laboratoriais relevantes, providenciar dados de acompanhamento prolongado e avaliar o impacto da terapia em complicações potenciais. A inclusão de métodos de análise de custo-efetividade deve ser considerada, sobretudo em tratamentos de longo prazo. A detecção de diferenças de tratamento em subgrupos relevantes, tais como crianças de diversas faixas etárias, deveria também ser considerada no cálculo de tamanho das amostras.

Com base nos estudos avaliados nesta metanálise, concluímos que a evidência-base para o uso de procinéticos no RGE e na DRGE em crianças é limitada, especialmente em lactentes, pois os poucos estudos se limitam a ensaios preliminares que avaliaram a resposta de curto prazo e com sérias limitações metodológicas, como baixa qualidade dos relatos, baixo poder estatístico e população heterogênea em termos de idade e ausência de medidas de desfecho de longo prazo. Portanto, há a necessidade de estudos com populações maiores, tipos de refluxo previamente definidos e desfechos pré-estabelecidos, empregando-se metodologia mais acurada, pois se trata de drogas amplamente utilizadas.

A probabilidade de resposta ao tratamento é significativamente maior para os procinéticos em relação ao placebo, com importante heterogeneidade para o grupo como um todo.

Em relação à resposta clínica, caracterizada por pelo menos alguma modificação nos sintomas, de curto prazo, a domperidona mostrou eficácia mais consistente do que a alcançada por outros procinéticos estudados devido ao número maior de crianças avaliadas. 


\section{Referências bibliográficas}

1. Vandenplas $\mathrm{Y}$, Ashkenazi A, Belli D, Boige N, Bouquet J, Cadranel $\mathrm{S}$ et al. A proposition for the diagnosis and treatment of gastro-oesophageal reflux disease in children: a report from a working group on gastro-oesophageal reflux disease. Working Group of the European Society of Paediatric Gastroenterology and Nutrition (ESPGAN). Eur J Pediatr 1993;152:704-11.

2. Orenstein SR, Izadnia F, Khan S. Gastroesophageal reflux disease in children. Gastroenterol Clin North Am 1999;28:947-69.

3. Rudolph CD, Mazur LJ, Liptak GS, Baker RD, Boyle JT, Colletti RB et al. Guidelines for evaluation and treatment of gastroesophageal reflux in infants and children: recommendations of the North American Society for Pediatric Gastroenterology and Nutrition. J Pediatr Gastroenterol Nutr 2001;32:S1-31.

4. Jung AD. Gastroesophageal reflux in infants and children. Am Fam Physician 2001;64:1853-60.

5. Chicella MF, Batres LA, Heesters MS, Dice JE. Prokinetic drug therapy in children: a review of current options. Ann Pharmacother 2005;39:706-11.

6. Vandenplas Y, Belli D, Benhamou P, Cadranel S, Cezard JP, Cucchiara S et al. A critical appraisal of current management practices for infant regurgitation - recommendations of a working party. Eur J Pediatr 1997;156:343-57.

7. Vandenplas Y, Belli DC, Benatar A, Cadranel S, Cucchiara S, Dupont C et al. The role of cisapride in the treatment of pediatric gastroesophageal reflux. The European Society of Paediatric Gastroenterology, Hepatology and Nutrition. J Pediatr Gastroenterol Nutr 1999;28:518-28.

8. Vandenplas Y, ESPGHAN Cisapride Panel, European Society for Pediatric Gastroenterology, Hepatology and Nutrition. Current pediatric indications for cisapride. J Pediatr Gastroenterol 2000;31:480-9.

9. Sandritter T. Gastroesophageal reflux disease in infants and children. J Pediatr Health Care 2003;17:198-205.

10. Hassall E. Decisions in diagnosing and managing chronic gastroesophageal reflux disease in children. J Pediatr 2005;146(Suppl 3):S3-12.

11. Pritchard DS, Baber N, Stephenson T. Should domperidone be used for the treatment of gastrooesophageal reflux in children? Systematic review of randomized controlled trials in children aged 1 month to 11 years old. $\mathrm{Br} \mathrm{J}$ Clin Pharmacol 2005;59:725-9.

12. Craig WR, Hanlon-Dearman A, Sinclair C, Taback S, Moffatt M. Metoclopramide, thickened feedings, and positioning for gastro-oesophageal reflux in children under two years (Cochrane Review). In: The Cochrane Library, Issue 1, 2006. Oxford: Update Software; 2006.

13. Petitti DB. Meta-analysis, decision analysis, and cost-effectiveness analysis: methods for quantitative synthesis in medicine. 2nd ed. New York: Oxford University Press, 2000.

14. Clarke M, Oxman AD, editors. Cochrane reviewers' handbook 4.1.6. [Updated January 2003]. In: The Cochrane Library. The Cochrane Collaboration. Oxford: Update Software; 2003.

15. Jadad AR, Moore RA, Carroll D, Jenkinson C, Reynolds DJ, Gavaghan DJ et al. Assessing the quality of reports of randomized clinical trials: is blinding necessary? Control Clin Trials 1996;17:1-12.
16. Freemantle N, Mason J, Eccles M. Deriving treatment recommendations from evidence within randomized trials. The role and limitation of meta-analysis. Int J Technol Assess Health Care 1999;15:304-15.

17. DerSimonian R, Laird N. Meta-analysis in clinical trials. Control Clin Trials 1986;7:177-88.

18. Higgins JP, Thompson SG, Deeks JJ, Altman DG. Measuring inconsistency in meta-analyses. BMJ 2003;327:557-60.

19. Orenstein SR. Tests to assess symptoms of gastroesophageal reflux in infants and children. J Pediatr Gastroenterol Nutr 2003;37:S29-32.

20. Bellissant E, Duhamel JF, Guillot M, Pariente-Khayat A, Olive G, Pons G. The triangular test to assess the efficacy of metoclopramide in gastroesophageal reflux. Clin Pharmacol Ther 1997;61:377-84.

21. Carroccio A, lacono G, Montalto G, Cavataio F, Soresi M, Notarbartolo A. Domperidone plus magnesium hydroxide and aluminum hydroxide: a valid therapy in children with gastroesophageal reflux. A double-blind randomized study versus placebo. Scand J Gastroenterol 1994;29:300-4.

22. Clara R. Chronic regurgitation and vomiting treated with domperidone. A multicenter evaluation. Acta Paediatr Belg 1979;32:203-7.

23. Cucchiara S, Staiano A, Corazziari E, Andreotti M, Auricchio S. A doubleblind controlled trial of domperidone in gastroesophageal reflux and peptic esophagitis in infancy. Gastroenterology 1985;88:1357.

24. De Loore I, Van Ravensteyn H, Ameryckx L. Domperidone drops in the symptomatic treatment of chronic pediatric vomiting and regurgitation. A comparison with metoclopramide. Postgrad Med J 1979;55:40-2.

25. Euler AR. Use of bethanechol for the treatment of gastroesophageal reflux. $J$ Pediatr 1980;96:321-4.

26. Bines JE, Quinlan JE, Treves S, Kleinman RE, Winter HS. Efficacy of domperidone in infants and children with gastroesophageal reflux. J Pediatr Gastroenterol Nutr 1992;14:400-5

27. Machida HM, Forbes DA, Gall DG, Scott RB. Metoclopramide in gastroesophageal reflux of infancy. J Pediatr 1988;112:483-7.

28. Leung AK, Lai PC. Use of metoclopramide for the treatment of gastroesophageal reflux in infants and children. Curr Ther Res Clin Exp 1984;36:911-5.

29. Ogorek CP, Fisher RS. Detection and treatment of gastroesophageal reflux disease. Gastroenterol Clin North Am 1989;18:293-313.

30. Carroll AE, Garrison MM, Christakis DA. A systematic review of nonpharmacological and nonsurgical therapies for gastroesophageal reflux in infants. Arch Pediatr Adolesc Med 2002;156:109-13.

31. Fischer M, Jesau R, Tinhof W. Management of gastrointestinal diseases using a motility-regulating preparation. Results of a field study using bromopride (Viaben) conducted by 530 general practitioners on 4182 patients. Fortschr Med 1979;97:883-8.

32. Barone JA. Domperidone: a peripherally acting dopamine2-receptor antagonist. Ann Pharmacother 1999;33:429-40.

33. Collins KK, Sondheimer JM. Domperidone-induced QT prolongation: add another drug to the list. J Pediatr 2008;153:596-8. 